\title{
Impact of the immune response modification by lysophosphatidylcholine in the efficacy of antibiotic therapy of experimental model of peritoneal sepsis and pneumonia by Pseudomonas aeruginosa
}

\author{
Raquel Parra-Millán 1,2*, Manuel Enrique Jiménez-Mejías 1,2\#, Rafael Ayerbe-Algaba ${ }^{1,2}$, Juan \\ Domínguez-Herrera ${ }^{1,2}$, Caridad Díaz ${ }^{3}$, José Pérez del Palacio ${ }^{3}$, Jerónimo Pachón ${ }^{2,4}$ and Younes \\ Smani ${ }^{1,2 *}$ \\ 1 Clinical Unit of Infectious Diseases, Microbiology and Preventive Medicine, University Hospital Virgen del \\ Rocío, 41013 Seville, Spain; rakel_rachel88@hotmail.com (R.P.M.); mej-mejias@telefonica.net (M.E.J.M); \\ ayerberafael@gmail.com (R.A.A.); judoher@yahoo.es (J.D.H.) \\ 2 Institute of Biomedicine of Seville (IBiS), University Hospital Virgen del Rocío, CSIC, University of Seville, \\ 41013 Seville, Spain; pachon@us.es (J.P.). \\ 3 Fundación Centro De Excelencia en Investigación de Medicamentos Innovadores en Andalucía, Fundación \\ MEDINA, Granada, Spain; caridad.diaz@medinaandalucia.es (C.D.); \\ jose.perezdelpalacio@medinaandalucia.es (J.P.d.P.) \\ 4 Department of Medicine, University of Seville, 41009 Seville, Spain \\ * Correspondence: ysmani-ibis@us.es; Tel.: +34-955-923-100 \\ \# These authors contributed equally to this work
}

\begin{abstract}
Immune response stimulation may be an adjuvant to antimicrobial treatment. Here, we evaluated the impact of immune response modification by lysophosphatidylcholine (LPC) combined with imipenem or ceftazidime in murine models of peritoneal sepsis (PS) and pneumonia by Pseudomonas aeruginosa. Imipenem and ceftazidime-susceptible strain (Pa39), and imipenem and ceftazidime-resistant strain (Pa238) were used. Ceftazidime pharmacokinetic and pharmacodynamic parameters were determined. Therapeutic efficacy, and TNF- $\alpha$ and IL-10 levels were determined in murine models of PS and pneumonia by Pa39 and Pa238 treated with LPC, imipenem or ceftazidime, alone or in combination. In PS model, LPC+ceftazidime reduced spleen and lungs Pa238 concentrations $\left(-3.45\right.$ and $\left.-3.56 \log _{10} \mathrm{CFU} / \mathrm{g} ; P<0.05\right)$ than ceftazidime monotherapy, while LPC+imipenem maintained the imipenem efficacy ( -1.66 and $\left.-1.45 \log _{10} \mathrm{CFU} / \mathrm{g} ; P>0.05\right)$. In pneumonia model, LPC+ceftazidime or LPC+imipenem reduced lungs Pa238 concentrations (-2.37 $\log _{10} \mathrm{CFU} / \mathrm{g}, P=0.1$, or $\left.-1.35 \log _{10} \mathrm{CFU} / \mathrm{g}, P=0.75\right)$. For Pa39 no statistically significant difference has been observed in PS and pneumonia models between combined therapy and monotherapy. Moreover, LPC+imipenem and LPC+ceftazidime decreased and increased significantly TNF- $\alpha$ and IL-10 levels, respectively, in comparison with untreated controls and monotherapies. These results demonstrate the impact of immune response modification by LPC plus antibiotics on the prognosis of infections by ceftazidime-resistant $P$. aeruginosa.
\end{abstract}

Keywords: Lysophosphatidylcholine; combined antimicrobial treatment; immune response; peritoneal sepsis model; pneumonia model; Pseudomonas aeruginosa.

\section{Introduction}

Pseudomonas aeruginosa, an ubiquitous microorganism, is one of the most relevant pathogens causing human opportunistic infections [1]. P. aeruginosa is a leading cause of severe nosocomial infections, particularly in critically ill and immunocompromised patients [2,3]. Indeed, $P$. aeruginosa is the top pathogen causing ventilator-associated pneumonia and burn wound infections and is a 
major cause of nosocomial bacteremia [2-4]. During the last decade, this pathogen has become increasingly resistant to most antimicrobials, including imipenem and ceftazidime [5].

A MDR pattern is commonly observed for $P$. aeruginosa clinical isolates, raising the threat of difficult-to-treat infections [6-8]. These MDR isolates are generally susceptible to polymyxins (colistin and polymyxin B) and resistant to imipenem and ceftazidime [9]. In a study which includes bacteremic patients by $P$. aeruginosa, ceftazidime and imipenem resistance rates were $36.6 \%$ and $22.8 \%$, respectively, and a multivariate analysis showed that resistance to both antimicrobial agents is a significant factor associated with mortality [10].

The retreat of the pharmaceutical sector from new antibiotic development, although, recently new beta-lactamases inhibitors against specific carbapenemases are developed and their clinical efficacy remains to be demonstrated in clinical trials [11], has exacerbated the challenge of widespread resistance and signals a critical need for innovation such as non-antimicrobial approaches and repurposing drugs. All these reasons have made necessary the urgent search for new alternatives for the treatment and control of infections by P. aeruginosa [5,6]. Not killing bacteria but avoiding the infection produced by P. aeruginosa, either immunizing the host or blocking the bacterial virulence factors, could be adjuvant approaches to reach new therapeutic goals.

The immune system stimulation by lysophosphatidylcholine (LPC) is one of the promising approaches. LPC is a major component of phospholipids involved in the recruitment and the stimulation of immune cells, and elimination of prokaryotic cells during infection [12-14]. We have demonstrated successfully the efficacy of LPC at $25 \mathrm{mg} / \mathrm{kg}$ as pre-emptive treatment in monotherapy and in combination with colistin, tigecycline or imipenem in murine peritoneal sepsis and pneumonia experimental models caused by susceptible and MDR Acinetobacter baumannii $[15,16]$.

In this study, we aimed to evaluate the impact of immune response modification by LPC in combination with imipenem or ceftazidime in murine models of peritoneal sepsis and pneumonia by susceptible (Pa39) and MDR (Pa238) P. aeruginosa strains.

\section{Results}

\subsection{Antimicrobial susceptibilities}

The MICs of imipenem, ceftazidime and LPC for Pa39 strain were 1, 1, and >8000 mg/L, respectively. The MICs of imipenem, ceftazidime, and LPC for Pa238 were 32, 64, and >8000 mg/L, respectively.

\subsection{Pharmacokinetic and pharmacodynamic parameters}

The pharmacokinetic and pharmacodynamic data for total imipenem and free ceftazidime are shown in Table S1.

\subsection{MLD100, LD50, and LD0 of P. aeruginosa}

To determine the MLD100, LD50, and LD0 of Pa39 and Pa238 strains, the murine peritoneal sepsis and pneumonia models were used. In the peritoneal sepsis, the mortality was dependent on the concentration of bacteria in the inoculum (data not shown). The MLD100, LD50, and LD0 of Pa39 strain were 3.85, 2.57 and $<1.81 \log _{10} \mathrm{CFU} / \mathrm{mL}$, respectively, and the MLD100, LD50, and LD0 of Pa238 strain were $6.7,4.65$ and $<3.08 \log _{10} \mathrm{CFU} / \mathrm{mL}$. With respect to the murine pneumonia model, the inoculum of both strains was concentrated to $10 \log _{10} \mathrm{CFU} / \mathrm{mL}$ for each strain to reach $100 \%$ of mice mortality, meanwhile the LD0 was $9 \log _{10} \mathrm{CFU} / \mathrm{mL}$.

\subsection{Efficacy of LPC and LPC combined treatments in murine peritoneal sepsis model}

The efficacy of LPC alone and in combination with imipenem or ceftazidime in murine peritoneal sepsis model after inoculation with MLD100 of Pa39 or Pa238 strains is shown in figure 1 and table 1. All treatments, except LPC alone, increased survival compared with the untreated controls for Pa39 strain $(P<0.05)$. For Pa238 strain, only LPC plus imipenem increased mice survival 
compared with the untreated controls $(P<0.05)$ (figure 1$)$. Regarding the spleen and lungs bacterial loads, imipenem monotherapy decreased the bacterial loads in $\approx 7.55 \log _{10} \mathrm{CFU} / \mathrm{g}(P<0.05)$, for Pa39 strain, and in 0.5 and $1.5 \log _{10} \mathrm{CFU} / \mathrm{g}$, respectively, for Pa238 strain, compared with the untreated controls. LPC plus imipenem decreased bacterial loads in both tissues in $\approx 8 \log _{10} \mathrm{CFU} / \mathrm{g}(P<0.05)$, for Pa39 strain, and in $\approx 2 \log _{10} \mathrm{CFU} / \mathrm{g}$, for Pa238 strain, compared with the untreated controls or LPC monotherapy. Moreover, ceftazidime monotherapy decreased the spleen and lungs bacterial loads in $\approx 7.5$ and $\approx 7.2 \log _{10} \mathrm{CFU} / \mathrm{g},(P<0.05)$, respectively, for Pa39 strain, and in 0.5 and $1.5 \log _{10} \mathrm{CFU} / \mathrm{g}$ $(P<0.05)$, respectively, for Pa238 strain, compared with the untreated controls. LPC plus ceftazidime decreased spleen and lungs bacterial loads in $\approx 8 \log _{10} \mathrm{CFU} / \mathrm{g}(P<0.05)$, for Pa39 strain, and in $\approx 4.5$ and $\approx 5 \log _{10} \mathrm{CFU} / \mathrm{g}(P<0.05)$, respectively, for Pa238 strain compared with the untreated controls or LPC monotherapy (Table 1). With respect to the bacteremia by Pa39 strain, imipenem and ceftazidime monotherapies reduced it to $\% \%$ compared with the untreated controls. For Pa238 strain, only LPC plus imipenem and LPC plus ceftazidime reduced the bacteremia to $93.33 \%$ and $53.33 \%(P<0.05)$, respectively, compared with the untreated controls (Table 1).
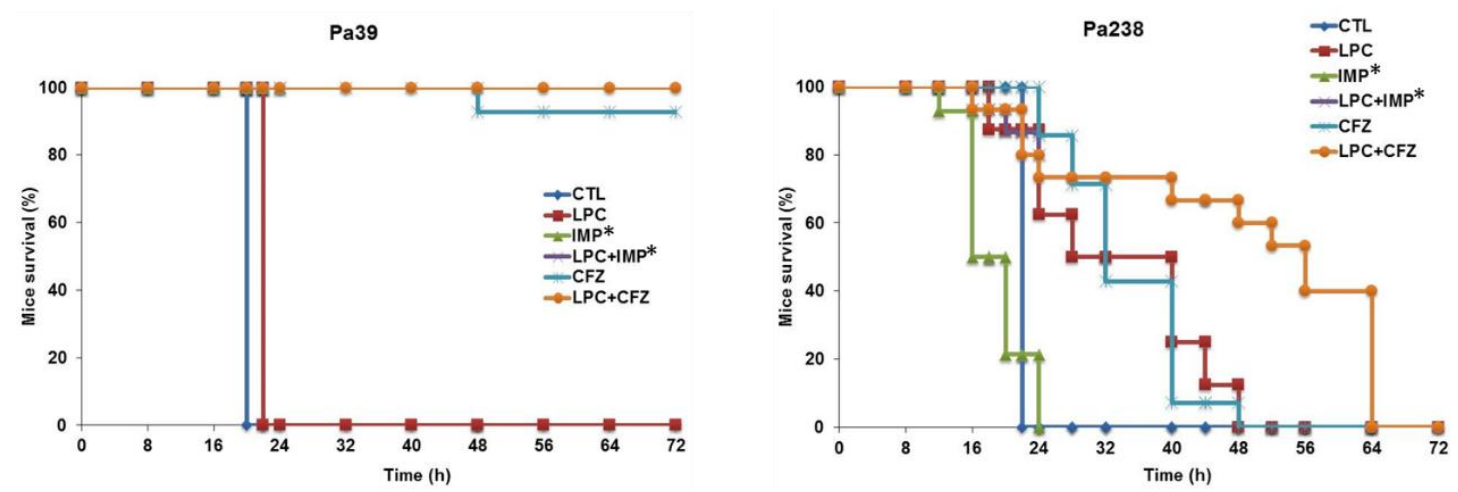

Figure 1. Mice survival after treatment with LPC in combination with imipenem or ceftazidime in peritoneal sepsis model by P. aeruginosa Pa39 and Pa238 strains. CTL, control (no treatment); LPC, lysophosphatidylcholine; IMP, imipenem; CFZ, ceftazidime. *: mice mortality was recorded over $24 \mathrm{~h}$ in imipenem w/o LPC

Table 1. Therapeutic effect of LPC in combination with imipenem or ceftazidime in murine peritoneal sepsis model of $P$. aeruginosa.

\begin{tabular}{|c|c|c|c|c|c|}
\hline \multirow{7}{*}{ Pa39 } & Treatment & $\mathbf{N}$ & $\begin{array}{c}\log _{10} \text { CFU/g of spleen } \\
(\text { mean } \pm \text { SEM) }\end{array}$ & $\begin{array}{c}\log _{10} \text { CFU/g of lungs } \\
(\text { mean } \pm \text { SEM })\end{array}$ & $\begin{array}{l}\text { Positive blood } \\
\text { cultures (\%) }\end{array}$ \\
\hline & CTL & 8 & $8.04 \pm 0.06$ & $7.78 \pm 0.10$ & 100 \\
\hline & LPC & 8 & $8.57 \pm 0.09^{\mathrm{a}}$ & $7.89 \pm 0.07$ & 100 \\
\hline & IMP & 14 & $0.54 \pm 0.29^{a, b}$ & $0.14 \pm 0.14^{\mathrm{a}, \mathrm{b}}$ & $0^{a, b}$ \\
\hline & LPC + IMP & 15 & $0.17 \pm 0.17^{\mathrm{a}, \mathrm{b}}$ & $0.14 \pm 0.14^{\mathrm{a}, \mathrm{b}}$ & $0^{a, b}$ \\
\hline & $\mathrm{CFZ}$ & 14 & $0.49 \pm 0.49^{a, b}$ & $0.49 \pm 0.49^{a, b}$ & $0^{a, b}$ \\
\hline & $\mathrm{LPC}+\mathrm{CFZ}$ & 15 & $0^{a, b}$ & $0^{a, b}$ & $0^{a, b}$ \\
\hline \multirow{2}{*}{$\mathrm{Pa} 238$} & CTL & 8 & $8.91 \pm 0.29$ & $8.42 \pm 0.35$ & 100 \\
\hline & LPC & 8 & $8.68 \pm 0.09$ & $8.06 \pm 0.21$ & 100 \\
\hline
\end{tabular}




\begin{tabular}{ccccc} 
IMP & 14 & $8.40 \pm 0.11$ & $7.78 \pm 0.10$ & 100 \\
\hline LPC + IMP & 15 & $6.74 \pm 0.74$ & $6.33 \pm 0.7$ & 100 \\
CFZ & 14 & $7.67 \pm 0,211_{a, b}$ & $6.90 \pm 0.24 a, b$ & $53^{a, b, c}$ \\
LPC + CFZ & 15 & $4.22 \pm 0.58^{\mathrm{a}, \mathrm{b}, \mathrm{c}}$ & $3.34 \pm 0.59 \mathrm{a}, \mathrm{b}, \mathrm{c}$ &
\end{tabular}

CTL, untreated controls (no treatment); LPC, lysophosphatidylcholine; IMP, imipenem; CFZ, ceftazidime. a: compared to the untreated controls, $P<0.05$; b: compared to LPC, $P<0.05$, c: compared to the IMP or CFZ, $P<0.05$.concentration that has been shown to be effective for $\geq 50 \%$ and $\geq 90 \%$ of isolates tested, respectively

\subsection{Efficacy of LPC and LPC combined treatments in murine experimental model of pneumonia}

The efficacy of LPC alone and in combination with imipenem or ceftazidime was tested in the murine pneumonia model after inoculation of $10 \log _{10}$ CFU/mL (MLD100) of each strain (Figure 2 and table 2). For both strains, treatment with LPC plus imipenem or LPC plus ceftazidime reduced mortality to $7 \%-64 \%$ compared with the untreated controls (figure 2). Regarding the bacterial lungs load, imipenem or ceftazidime decreased Pa39 and Pa238 strains by $4.87(P<0.05)$ and $1.08 \log _{10}$ CFU/g or $4.48(P<0.05)$ and $1.26 \log _{10} \mathrm{CFU} / \mathrm{g}$, respectively, compared with the untreated controls. LPC plus imipenem decreased bacterial lungs load of Pa39 and Pa238 strains by 5.91 and $4.89 \log _{10}$ CFU/g $(P<0.05)$, or $4.88(P<0.05)$ and $2.79 \log _{10} \mathrm{CFU} / \mathrm{g}$, compared with the untreated controls or LPC monotherapy. LPC plus ceftazidime decreased bacterial lungs load of Pa39 and Pa238 strains by 5.02 $(P<0.05)$ and $3.63 \log _{10} \mathrm{CFU} / \mathrm{g}$, or in $3.99(P<0.05)$ and $0.49 \log _{10} \mathrm{CFU} / \mathrm{g}$, respectively, compared with the untreated controls or LPC monotherapy (Table 2). With respect to bacteremia, LPC plus imipenem and LPC plus ceftazidime reduced it by both strains to $\approx 50 \%-93 \%$ compared with the untreated controls, and to $\approx 20 \%-40 \%$ compared with the antimicrobial monotherapies $(P<0.05)($ Table 2).
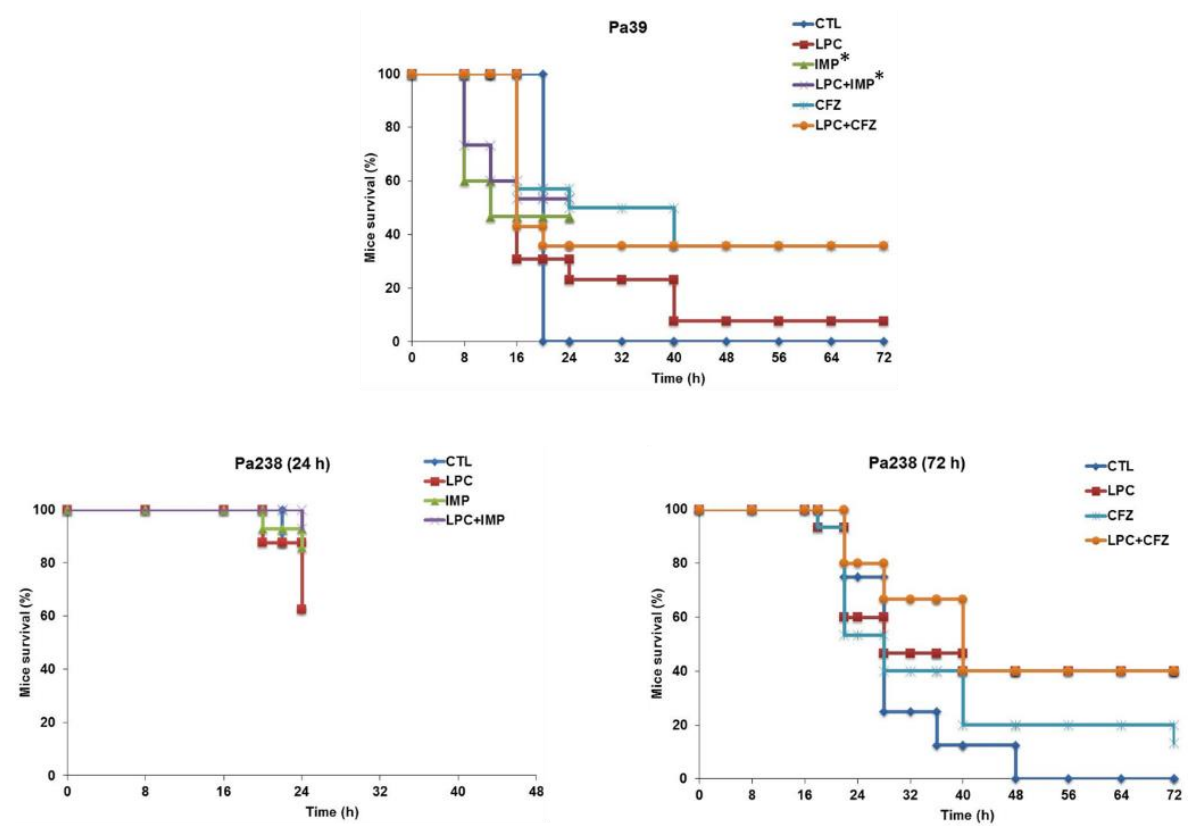

Figure 2. Mice survival after treatment with LPC in combination with imipenem or ceftazidime in pneumonia model by P. aeruginosa Pa39 and Pa238 strains. CTL, control (no treatment); LPC, lysophosphatidylcholine; IMP, imipenem; CFZ, ceftazidime. *: mice mortality was recorded over $24 \mathrm{~h}$ in imipenem w/o LPC. 
Table 2. Therapeutic effect of LPC in combination with imipenem or ceftazidime in murine pneumonia model of $P$. aeruginosa.

\begin{tabular}{|c|c|c|c|c|}
\hline & Treatment & $\mathbf{N}$ & $\begin{array}{c}\log _{10} \text { CFU/g of lungs } \\
(\text { mean } \pm \text { SEM })\end{array}$ & $\begin{array}{c}\text { Positive blood culture } \\
\qquad(\%)\end{array}$ \\
\hline \multirow{6}{*}{ Pa39 } & CTL & 8 & $9.44 \pm 0.19$ & 100 \\
\hline & LPC & 13 & $8.41 \pm 0.72$ & 92 \\
\hline & IMP & 15 & $4.57 \pm 0.56 \mathrm{a}, \mathrm{b}$ & $47_{a, b}$ \\
\hline & $\mathrm{LPC}+\mathrm{IMP}$ & 15 & $3.53 \pm 0.60_{a, b}$ & $7 \mathrm{a}, \mathrm{b}, \mathrm{c}$ \\
\hline & $\mathrm{CFZ}$ & 14 & $4.96 \pm 0.93 \mathrm{a}, \mathrm{b}$ & 64 \\
\hline & $\mathrm{LPC}+\mathrm{CFZ}$ & 14 & $4.42 \pm 0.93 \mathrm{a}, \mathrm{b}$ & $43_{a, b}$ \\
\hline \multirow{4}{*}{$\begin{array}{l}\mathrm{Pa} 238 \\
(24 \mathrm{~h})\end{array}$} & CTL & 8 & $8.38 \pm 0.73$ & 100 \\
\hline & LPC & 8 & $8.74 \pm 0.83$ & 100 \\
\hline & IMP & 14 & $7.30 \pm 0.68$ & $57 \mathrm{a}, \mathrm{b}$ \\
\hline & $\mathrm{LPC}+\mathrm{IMP}$ & 14 & $5.95 \pm 0.83 a$ & $21_{a, b}$ \\
\hline \multirow{4}{*}{$\begin{array}{l}\mathrm{Pa} 238 \\
(72 \mathrm{~h})\end{array}$} & CTL & 8 & $10.84 \pm 0.07$ & 100 \\
\hline & LPC & 15 & $7.7 \pm 1.11$ & 73 \\
\hline & CFZ & 15 & $9.58 \pm 0.53$ & 93 \\
\hline & $\mathrm{LPC}+\mathrm{CFZ}$ & 15 & $7.21 \pm 0.79 a$ & $53 \mathrm{a}, \mathrm{c}$ \\
\hline
\end{tabular}

CTL, untreated controls (no treatment); LPC, lysophosphatidylcholine; IMP, imipenem; CFZ, ceftazidime.

a: compared to the untreated controls, $P<0.05$; b: compared to the LPC, $P<0.05$; c: compared to the IMP or CFZ, $P<0.05$.

\subsection{Cytokines production}

The effects of different treatments on cytokine production, in peritoneal sepsis and pneumonia models by Pa238 strain were evaluated (figure 3). In the peritoneal sepsis model, imipenem and ceftazidime monotherapies increased non-significantly the release of TNF- $\alpha$ to $5207.61 \pm 2859.34$ $\mathrm{pg} / \mathrm{mL}$ and $5232.59 \pm 3905.02 \mathrm{pg} / \mathrm{mL}$, respectively, and decreased the release of IL-10 to $1002.25 \pm$ $405.43 \mathrm{pg} / \mathrm{mL}(P<0.05)$ and $761.96 \pm 182.09 \mathrm{pg} / \mathrm{mL}(P<0.05)$, respectively, at $8 \mathrm{~h}$ (time that correspond to $4 \mathrm{~h}$ of imipenem and ceftazidime treatment), compared with the untreated controls at $8 \mathrm{~h}: 1900.60$ $\pm 638.01 \mathrm{pg} / \mathrm{mL}$ for TNF- $\alpha$, and $2423.29 \pm 607.45 \mathrm{pg} / \mathrm{mL}$ for IL-10. Meanwhile, LPC plus imipenem and LPC plus ceftazidime combinations decreased the release of TNF- $\alpha$ to $62.7 \pm 14.3 \mathrm{pg} / \mathrm{mL}(P<0.05)$ and $63.53 \pm 19.42 \mathrm{pg} / \mathrm{mL}(P<0.05)$, respectively, and increased the release of IL-10 to $4950.25 \pm 202.67$ $\mathrm{pg} / \mathrm{mL}(P<0.05)$ and $3829.5 \pm 1760.76 \mathrm{pg} / \mathrm{mL}(P<0.05)$, respectively, at $8 \mathrm{~h}$ compared with the imipenem and ceftazidime monotherapies: $5207.61 \pm 2859.34 \mathrm{pg} / \mathrm{mL}$ and $5232.59 \pm 3905.02 \mathrm{pg} / \mathrm{mL}$, respectively, for TNF- $\alpha$, and $1002.25 \pm 405.43 \mathrm{pg} / \mathrm{mL}$ and $761.96 \pm 182.09 \mathrm{pg} / \mathrm{mL}$ for IL-10. In the case of IL-6, imipenem and ceftazidime monotherapies increased the release of IL-6 to $2274.4 \pm 113.51 \mathrm{pg} / \mathrm{mL}$ $(P=0.018)$ and $2148.58 \pm 3.4 .03 \mathrm{pg} / \mathrm{mL}(P=0.053)$, respectively, at $8 \mathrm{~h}$ compared with the control group: 
$748.2 \pm 362.84 \mathrm{pg} / \mathrm{mL}$. Meanwhile, LPC plus imipenem and LPC plus ceftazidime combinations induced similar release of IL-6 compared with imipenem and ceftazidime monotherapies (figure 3A).

In the pneumonia model, similar results of the effect of imipenem and ceftazidime monotherapies, LPC plus imipenem and LPC plus ceftazidime combinations on the serum TNF- $\alpha$ and IL-10 levels at $8 \mathrm{~h}$ post-bacterial inoculation were observed. LPC plus imipenem and LPC plus ceftazidime combinations decreased the release of TNF- $\alpha$ to $150.73 \pm 52.7 \mathrm{pg} / \mathrm{mL}(P<0.05)$ and $54.77 \pm$ $3.21 \mathrm{pg} / \mathrm{mL}(P<0.05)$, respectively, and increased the release of IL-10 to $1648 \pm 969.97 \mathrm{pg} / \mathrm{mL}(P<0.05)$ and $809.54 \pm 130.07 \mathrm{pg} / \mathrm{mL}(P<0.05)$, respectively, compared with the imipenem and ceftazidime monotherapies: $1007.17 \pm 267.19 \mathrm{pg} / \mathrm{mL}$ and $1564.55 \pm 101.84 \mathrm{pg} / \mathrm{mL}$, respectively, for TNF- $\alpha$, and $35.33 \pm 14.33 \mathrm{pg} / \mathrm{mL}$ and $44.75 \pm 7.7 \mathrm{pg} / \mathrm{mL}$, respectively, for IL-10. In the case of IL-6, imipenem and ceftazidime monotherapies increased non-significantly the release of IL-6 to $929.42 \pm 188.11 \mathrm{pg} / \mathrm{mL}$ and $1360.49 \pm 475.24 \mathrm{pg} / \mathrm{mL}$, respectively, at $8 \mathrm{~h}$ compared with the control group: $855.40 \pm 459.13$ $\mathrm{pg} / \mathrm{mL}$. Moreover LPC+imipenem and LPC+ceftazidime combinations increased the release of IL-6 to $3130.40 \pm 558.14 \mathrm{pg} / \mathrm{mL}(P=0.043)$ and $1884.62 \pm 68.13 \mathrm{pg} / \mathrm{mL}(P=0.212)$, respectively, at $8 \mathrm{~h}$ compared with imipenem and ceftazidime monotherapies: $929.42 \pm 188.11 \mathrm{pg} / \mathrm{mL}$ and $1360.49 \pm$ $475.24 \mathrm{pg} / \mathrm{mL}$, respectively (figure 3B).

Importantly, imipenem and ceftazidime treatments in healthy mice did not changed significantly the release of TNF- $\alpha$, IL-6, and IL-10 at 4 and $8 \mathrm{~h}$ when compared with mice without antibiotic treatments (figure 3 ).

A
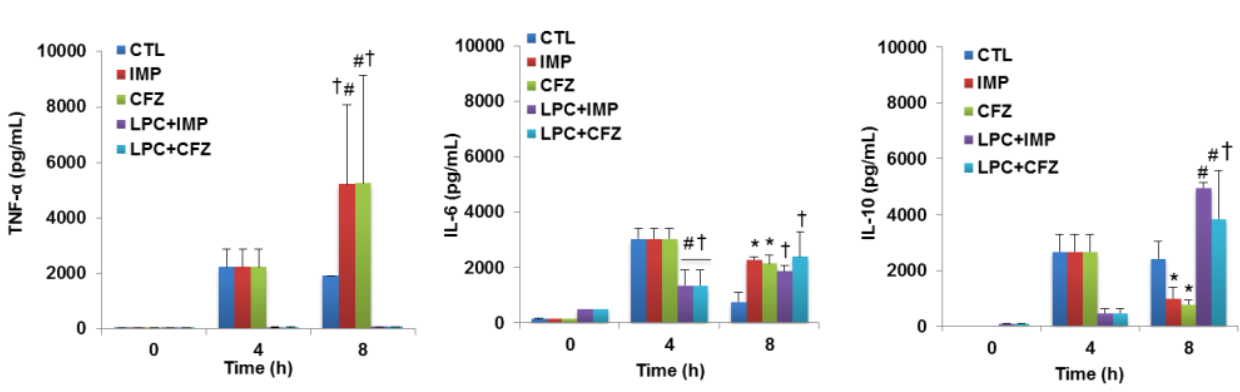

B
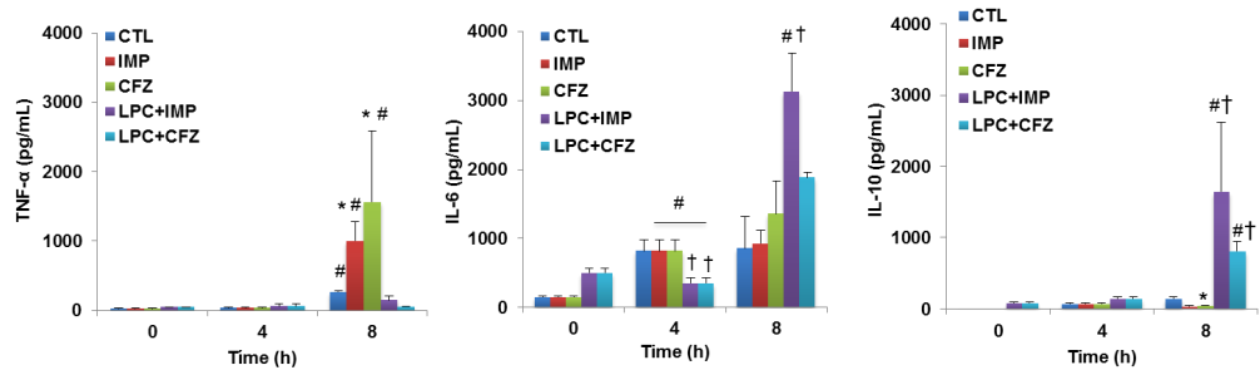

Figure 3. Cytokine production after MDR P. aeruginosa-induced murine peritoneal sepsis (A) and pneumonia (B) experimental models. Levels of TNF- $\alpha$, IL-6 and IL-10 in serum were determined from 0 to $8 \mathrm{~h}$ for mice inoculated with the Pa238 strain and treated or not with imipenem, ceftazidime, LPC-imipenem combination or LPC-ceftazidime combination. Representative results are shown, and the data are presented as means. CTL, untreated controls (no treatment); LPC, lysophosphatidylcholine; IMP, imipenem; CFZ, ceftazidime. * and + : compared to CTL, $P<0.05$; \#: compared to IMP or CFZ. $P<0.05$.

\section{Discussion}

This study shows that imipenem and ceftazidime monotherapies in both experimental models by the susceptible Pa39 strain and the MDR Pa238 strain reduced the bacterial tissues concentrations and bacteremia, and increased survival, in concordance with their antimicrobial activities. 
In the pneumonia model by the susceptible strain, treatment with LPC plus imipenem or LPC plus ceftazidime did not reduce significantly the bacterial lungs load, bacteremia, nor mortality, when compared with antimicrobial monotherapies, except bacteremia for imipenem. In the case of the MDR strain, as expected, no therapeutic effect was observed with imipenem or ceftazidime due to resistance to both antimicrobials; however, the treatment with LPC plus imipenem or LPC plus ceftazidime, reduced bacterial loads and bacteremia by $\approx 1.35-2.35 \log _{10} \mathrm{CFU} / \mathrm{g}$ and $50 \%$, respectively, compared with antimicrobial monotherapies; and survival increased slightly. Similar data has been observed with LPC plus imipenem or LPC plus tigecycline in the A. baumannii resistant to imipenem and tigecycline pneumonia model [16].

In the peritoneal sepsis model by the MDR Pa238 strain LPC plus ceftazidime did not improve survival at $72 \mathrm{~h}$, even if the bacterial burden in tissue was lower than in untreated controls and ceftazidime monotherapy; the analysis of survival at $24 \mathrm{~h}$ showed a mortality of $27 \%$ ( 4 out of 15 mice), mortality similar to the $20 \%$ at $24 \mathrm{~h}$ with LPC plus imipenem. This data suggests that LPC, administered in one dose previous to the inoculation, only reduced early mice mortality. In the same line of this result, in 2013 Jacqueline et al. demonstrated that in murine experimental model of pneumonia by $P$. aeruginosa, the bacterial burden in spleen and lung after treatment with ceftazidime was 2.74 and $4.74 \log _{10} \mathrm{CFU} / \mathrm{mL}$, and the mortality reached $80 \%$ [17].

It is important to note that ceftazidime in combination with LPC against MDR Pa238 strain in peritoneal sepsis model did not improve the mice survival than ceftazidime and LPC monotherapy, or control animal. Meanwhile, ceftazidime combined with LPC increased the mice survival in the pneumonia model. In the case of others Gram-negative bacilli such as A. baumannii ATCC 17978 strain, we found that the LPC monotherapy in peritoneal sepsis model only increased $40 \%$ of mice survival vs. the $68 \%$ observed in the pneumonia model [15]. In the same way, rifampicin combined with colistin did not improve the mice survival in peritoneal sepsis model by carbapenemaseproducing Klebsiella pneumoniae than in pneumonia model (unpublished data).

Furthermore, we showed that LPC monotherapy in peritoneal sepsis model by susceptible Pa39 strain and MDR Pa238 strain had no significant therapeutic effect. In contrast, in the pneumonia model by susceptible Pa39 and MDR Pa238 strains, we have observed that LPC monotherapy for 72 $\mathrm{h}$ reduced the bacterial loads in lungs by 1.03 and $3.14 \log _{10} \mathrm{CFU} / \mathrm{g}$, respectively. This difference in the results between both experimental models of infections would be due to the severity of peritoneal sepsis model which the sepsis was defined as the result of a dysregulated systemic inflammatory response syndrome in the presence of infection, accompanied by major organ failure and death [18]. This infection severity do not allowed LPC in monotherapy to reduce significantly the bacterial loads of both strains in tissues. Moreover, difference in the effect of LPC on both strains in the pneumonia model has been observed which was more present on the MDR Pa238 strain than on the susceptible Pa39 strain. This would be due to the difference in the virulence degree of both strains which the susceptible Pa39 strain caused 100\% of mice mortality in the first $24 \mathrm{~h}$ in contrast of the MDR strain that caused only $37 \%$ of mice mortality in the first $24 \mathrm{~h}$ of the pneumonia model.

For other pathogens such as Staphylococcus aureus, Miyazaki et al. showed in vitro that LPC can enhance the antimicrobial effects of gentamicin against methicillin-resistant S. aureus (MRSA); suggesting the application of LPC as a beneficial additive in topical antibiotics for superficial skin infections [19]. The mode of action of LPC is different following the pathogen species. In Gram positive bacteria, LPC can directly induce MRSA killing by interacting with cytoplasmic membrane and inducing membrane depolarization and increased membrane permeability [19]. In Gram negative bacteria, LPC had not direct effect on these bacteria due to their outer membrane preventing the interaction between LPC and bacterial cytoplasmic membrane $[15,16,20]$. The beneficial effects of LPC alone against E. coli have been associated with the activation of hydrogen peroxide by neutrophils and with the induction of phagocytosis by macrophages through the activation of AMPactivated protein kinase $[20,21]$. In LPC combination with antibiotics treatments against $P$. aeruginosa 
infections, these ways can be suggested as one of the mode of action of LPC to adjunct the antibiotics effect. Besides, LPC alone and in combination with antibiotics treatment against E. coli and $A$. baumannii have been previously associated with the modulation of inflammation such as upregulation of monocyte chemotactic protein-1, and pro- and anti-inflammatory cytokines release $[12,15,16,20]$. Interestingly, comparing the effect of the pro-inflammatory cytokine TNF- $\alpha$, the combination of LPC plus imipenem or LPC plus ceftazidime reduced significantly at $8 \mathrm{~h}$ postbacterial inoculation the TNF- $\alpha$ levels by 83- or 82-folds, respectively, in the peritoneal sepsis model by MDR strain. In contrast, these reductions were lower in the pneumonia model: 7 - or 28-folds with LPC plus imipenem or LPC plus ceftazidime, respectively. These differences in the anti-inflammatory effect of LPC in both models could be the cause of the different results in terms of mice survival. These data are in accordance with the previously reported immunomodulatory effects of LPC [15,20]. It is important to mention, that immune response developed in mice treated with LPC and ceftazidime and infected by Pa39 strain in peritoneal sepsis model can help to prevent the re-infection by the same strain 7 days after treatment stopping (data not shown). This data allowed us to suggest that LPC in combination with antibiotics would be able to induce immune response memory to prevent the reinfection. More studies are needed to decipher this effect.

Some studies have been already performed to control the infections by $P$. aeruginosa by the use of small peptides or molecules with immunomodulatory properties. Among them, [E6k,D9k] hymenochirin-1B [22], present high antibacterial activities and immunomodulatory properties in vitro. Other studies have evaluated peptides of the human innate immune system. Human betadefensins hBD-2, hBD-3, and hBD-4 have presented bactericidal activities against P. aeruginosa [2325]. LL-37, a cationic peptide of the cathelecidins family, exhibited significant antimicrobial activity against $P$. aeruginosa and prevented biofilm formation, adding significance to its efficacy [26,27].

In humans, the application of granulocyte colony-stimulating factor (G-CSF) as an adjuvant with antibiotics in patients with either community-acquired pneumonia or hospital-acquired pneumonia, and application of corticosteroids as an adjunct treatment of patients with community-acquired pneumonia were performed [28,29]. Positive results have been seen in animals for both applications, but when mixed with clinical results, G-CSF application does not mimic the results seen with the animal model [28].

As we demonstrated in this study, LPC both as preemptive therapy or in combination with antimicrobial agents has shown promising in vivo results in severe experimental models of infections by $A$. baumannii $[15,16]$ and $P$. aeruginosa. However, caution is needed and further extensive in vivo studies and clinical trials have to be performed to confirm the potential use of these adjuvants including LPC as true therapeutic alternatives.

The present study has the limitation that LPC was administered in only one dose, before the bacterial inoculation; thus, its immunomodulatory effect is probably in a reduced period. However, these results warrant performing additional studies to determine whether multiple doses of LPC in combination with antimicrobial agents, may modify the immune response for extended period and improve the effect of LPC in combination with antimicrobials against Gram-negative bacilli infections.

\section{Materials and Methods}

\subsection{Bacterial strains}

P. aeruginosa (Pa39) clinical strain susceptible to ceftazidime and imipenem isolated from blood culture, and MDR P. aeruginosa (Pa238) clinical strain resistant to ceftazidime, imipenem, ciprofloxacin and tobramycin, isolated from blood culture were used. Both strains were from the REIPI-GEIH 2008 collection [30]. 


\subsection{Antimicrobial agents and reagents}

For the in vitro assays, antimicrobials were used as standard laboratory powders: ceftazidime and imipenem (Sigma, Spain). For the in vivo experiments, clinical formulations of antimicrobials were used: ceftazidime (Normon, Spain) and imipenem (Merk Sharp \& Dohme, Spain). The anesthetic was 5\% (w/v) sodium thiopental administered intraperitoneally (i.p.) (B. Braun Medical S.A., Spain)..

\subsection{In vitro susceptibility testing}

Minimal inhibitory concentrations (MICs) were determined by broth microdilution assay according to standard CLSI recommendations [31] as previously described [32]. Escherichia coli ATCC 25922 was used as a control strain.

\subsection{Animals}

Immunocompetent C57BL/6 female mice, weighting 18 to $20 \mathrm{~g}$ (Production and Experimentation Animal Center, University of Seville, Seville, Spain) were used. Animals were housed in regulation cages and given free access to food and water. This study was carried out in strict accordance with the recommendations in the Guide for the Care and Use of Laboratory Animals [33]. The protocol was approved by the Committee of Ethics for Animal Experiments of the University Hospital of Virgen del Rocío of Seville (2014PI/014).

\subsection{Antimicrobial pharmacokinetics and pharmacodynamics parameters}

Serum LPC and imipenem levels were previously determined by our research group $[15,16]$. Serum ceftazidime levels were determined in groups of 30 healthy mice following single doses of 100 $\mathrm{mg} / \mathrm{kg}$ i.p. ceftazidime. In sets of three animals at $0,5,10,15,30,60,90,120,240,480$ and $1440 \mathrm{~min}$ after antimicrobial administration, blood samples were obtained from anesthetized mice from periorbital plexus. Concentrations of ceftazidime were measured using a HPLC-tandem mass spectrometry (LC-MS/MS) [34]. The Cmax in serum, AUC0- $\infty, \mathrm{t} 1 / 2$, and $\mathrm{T}>\mathrm{MIC}$ ratios were obtained by a computer-assisted method [35]. Final dosing of free ceftazidime in the in vivo experiments was adjusted to achieve a $>$ MIC at least $\approx 40 \%-50 \%$ of the dosing interval [36].

\subsection{Experimental murine model of peritoneal sepsis}

The previously characterized murine peritoneal sepsis model by A. baumannii was used [15]. Briefly, animals were inoculated i.p. with $0.5 \mathrm{~mL}$ of the minimal lethal dose 100 (MLD100) of Pa39 or Pa238 strains, mixed 1:1 with 10\% porcine mucin (Sigma, Spain). The MLD100, lethal dose 50 (LD50) and lethal dose $0\left(\mathrm{LD}_{0}\right)$ were determined by inoculating groups of 6 mice i.p. with decreasing concentrations of $P$. aeruginosa from 7.6 to $3.85 \mathrm{Log}$ CFU/mL for Pa39 strain, and from 7.8 to 4 Log $\mathrm{CFU} / \mathrm{mL}$ for Pa238 strain, and monitoring the survival of the mice for 7 days; these values were determined using the Probit method. LPC therapy was administered as a pretreatment $1 \mathrm{~h}$ before bacterial inoculation, and antimicrobial therapy was initiated $4 \mathrm{~h}$ after bacterial inoculation. Groups of 15 mice were randomly ascribed to the following groups: 1) untreated controls (without treatment), 2) LPC administered once at $75 \mathrm{mg} / \mathrm{kg}$ i.p. $1 \mathrm{~h}$ before bacterial inoculation, 3) ceftazidime administered i.p. at $100 \mathrm{mg} / \mathrm{kg} / 12 \mathrm{~h}$ for $72 \mathrm{~h}, 4$ ) imipenem administered i.m. at $30 \mathrm{mg} / \mathrm{kg} / 4 \mathrm{~h}$ for $24 \mathrm{~h}$, and 5 and 6) the combinations of LPC at $75 \mathrm{mg} / \mathrm{kg}$ and ceftazidime at $100 \mathrm{mg} / \mathrm{kg} / 12 \mathrm{~h}$, and imipenem at $30 \mathrm{mg} / \mathrm{kg} / 4 \mathrm{~h}$, respectively. The antimicrobial dosages were chosen after obtaining the PK/PD data.

Mortality was recorded over $24 \mathrm{~h}$ (for imipenem treatment groups) or $72 \mathrm{~h}$ (for ceftazidime treatment group). After the death or the putting down of the mice at the end of the experimental period, aseptic thoracotomies were performed, and blood samples were obtained by cardiac puncture for qualitative blood cultures. Samples were inoculated in sterile tubes with $1 \mathrm{~mL}$ of Luria Bertani 
(LB) broth and incubated for $24 \mathrm{~h}$ at $37^{\circ} \mathrm{C}$, and then $100 \mu \mathrm{L}$ were plated onto sheep blood agar. The results of the blood cultures are expressed as positive (when $\geq 1$ CFU was present in the plate) or negative. The spleen and lungs were aseptically removed and homogenized (Stomacher 80; Tekmar Co., USA) in $2 \mathrm{~mL}$ of sterile $\mathrm{NaCl} 0.9 \%$ solution. Ten-fold dilutions of the homogenized spleen and lungs were plated onto sheep blood agar for quantitative cultures ( $\log _{10} \mathrm{CFU} / \mathrm{g}$ of spleen or lung).

\subsection{Experimental murine model of pneumonia}

A previously described experimental murine pneumonia model [37] was used to evaluate the efficacy of LPC in monotherapy and in combination with antimicrobial agents against Pa39 and Pa238 strains. Briefly, the mice were anesthetized by an i.p. injection of 5\% (wt/vol) sodium thiopental. They were suspended vertically, and the trachea of each was then cannulated with a blunt-tipped metal needle. The fell of the needle tip against the tracheal cartilage confirmed the intratracheal location. A microliter syringe (Hamilton Co., Reno, NV) was used for inoculation of $50 \mu \mathrm{L}$ of the MLD100.

The mice remained in a vertical position for $3 \mathrm{~min}$ and then in a $30^{\circ}$ position until awake. The MLD100 and LD0 were determined by inoculating groups of 6 mice intratracheally with decreasing concentrations of Pa39 and Pa238 strains from 10 to $9 \log _{10} \mathrm{CFU} / \mathrm{mL}$, and monitoring the survival of the mice for 7 days. Treatment groups were similar to the experimental model of peritoneal sepsis. After death or putting down of the mice at the end of the experimental period, aseptic thoracotomies were performed, and blood samples for qualitative blood culture were obtained by cardiac puncture (data are reported as numbers [\%] of positive cultures). The lungs were aseptically removed and homogenized as described above for quantitative culture (data are reported in $\log _{10}$ CFU/g of lung).

\subsection{Cytokine assay}

Blood samples were collected from the periorbital plexuses of 72 anesthetized mice infected or not with the Pa238 at the MLD100 in the peritoneal sepsis and pneumonia models and treated or not with imipenem, ceftazidime, LPC-imipenem combination or LPC-ceftazidime combination, as previously described [16]. Serum levels of tumor necrosis factor alpha (TNF- $\alpha$ ), interleukin-6 (IL-6) and interleukin-10 (IL-10) were determined in mice at 0,4 , and $8 \mathrm{~h}$ post-infection or treatment with imipenem or ceftazidime by using an enzyme-linked immunosorbent assay (ELISA) (eBioscience, Spain) following the protocol presented in the figure S1.

\subsection{Statistical analysis}

Differences in bacterial spleen and lungs concentrations (mean \pm standard error of the mean (SEM) $\log$ CFU/g of tissue) were assessed by analysis of variance (ANOVA) and post-hoc Dunnett test. Differences in blood sterility (\%) between groups were compared by $\chi 2$ test after normalization determination by Kolmogorov-Smirnov test. For mice survival model, a Kaplan-Meier test was performed to determine the difference between mortality rates. A $P$-value of $<0.05$ was considered significant. The SPSS (version 17.0) statistical package was used (SPSS Inc.).

\section{Conclusions}

The present study suggests that LPC in combination with ceftazidime or imipenem, in murine peritoneal sepsis and pneumonia models by clinical MDR P. aeruginosa isolate, tends to improve the antibacterial activity of ceftazidime and imipenem by reducing bacterial tissue and blood concentrations and increasing mice survival..

Author Contributions: Y.S. conceived the study and designed the experiments, analyzed the results and wrote the manuscript. R.P.-M., R.A.-A, J.D.-H., C.D. and J.P.-P. carried out the experiments. R.P.-M. and R.A.-A. analyzed the data. M.E.J.-M. and J.P. revised the manuscript. 
Funding: This work was supported by Instituto de Salud Carlos III, Subdirección General de Redes y Centros de Investigación Cooperativa, Ministerio de Ciencia e Innovación (PI13/01744, PI16/01306), and by Plan Nacional de I+D+i 2013-2016 and Instituto de Salud Carlos III, Subdirección General de Redes y Centros de Investigación Cooperativa, Ministerio de Ciencia e Innovación, Spanish Network for Research in Infectious Diseases (RD16/0016/0009), cofinanced by the European Development Regional Fund “A Way to Achieve Europe," Operative Program Intelligent Growth 2014-2020. Y.S. is supported by the Subprograma Miguel Servet Tipo I, Instituto de Salud Carlos III, Subdirección General de Redes y Centros de Investigación Cooperativa, Ministerio de Ciencia e Innovación, Spain [CP15/00132]. The MEDINA authors disclosed the receipt of financial support from Fundación MEDINA, a public-private partnership of Merck Sharp \& Dohme de España S.A./Universidad de Granada/Junta de Andalucía.

Acknowledgments: We would like to thank Dr. Antonio Oliver for the kind gift of the Pseudomonas aeruginosa strains Pa39 and Pa238.

Part of this study was presented at the 27th European Congress of Clinical Microbiology and Infectious Diseases, Vienna, Austria 2017.

Conflicts of Interest: The authors declare that the research was conducted in the absence of any commercial or financial relationships that could be construed as a potential conflict of interest.

\section{References}

1. Gellatly, S.L.; Hancock, R.E.W. Pseudomonas aeruginosa: New insights into pathogenesis and host defenses. Pathog. Dis. 2013, 67, 159-173.

2. Kang, C.I.; Kim, S.H.; Kim, H.B.; Park, S.W.; Choe, Y.J.; Oh, M.D.; Kim, E.C.; Choe, K.W. Pseudomonas aeruginosa Bacteremia: Risk factors for mortality and influence of delayed receipt of effective antimicrobial therapy on clinical outcome . Clin. Infect. Dis. 2003, 37, 745-751.

3. Vidal, F.; Mensa, J.; Almela, M.; Martínez, J.A.; Marco, F.; Casals, C., Gatell, J.M..; Soriano, E.; Jiménez, de Anta, M.T. Epidemiology and outcome of Pseudomonas aeruginosa bacteremia, with special emphasis on the influence of antibiotic treatment: Analysis of 189 episodes. Arch. Intern. Med. 1996, 156, 2121 2126.

4. Vincent, J.L. Nosocomial infections in adult intensive-care units. Lancet 2003, 361, 2068-2077.

5. European Centre for Disease Prevention and Control. Annual epidemiological report 2014. Antimicrobial resistance and healthcare-associated infections. Ecdc http://ecdc.europa.eu/en/publications/Publications/antimicrobial-resistance-annual-epidemiologicalreport.pdf. Accessed 01 July 2016 (2014) doi:10.2903/j.efsa.2015.4329.

6. Ali, Z.; Mumtaz, N.; Naz, S.A.; Jabeen, N.; Shafique, M. Multi-Drug Resistant Pseudomonas aeruginosa: A threat of nosocomial infections in tertiary care hospitals. J. Pak. Med. Assoc. 2015, 65(1), 12-16.

7. Micek, S.T.; Wunderink, R.G.; Kollef, M.H.; Chen, C.; Rello, J.; Chastre, J., Antonelli, M.; Welte, T.; Clair, B.; Ostermann, H.; Calbo, E.; Torres, A.; Menichetti, F.; Schramm, G.E.; Menon, V. An international multicenter retrospective study of Pseudomonas aeruginosa nosocomial pneumonia: Impact of multidrug resistance. Crit. Care 2015, 19, 219.

8. Kaye, K.S.; Pogue, J.M. Infections Caused by Resistant Gram-Negative Bacteria: Epidemiology and Management. Pharmacotherapy 2015, 35, 949-962.

9. El Solh, A.A.; Alhajhusain, A. Update on the treatment of Pseudomonas aeruginosa pneumonia. J. Antimicrob. Chemother. 2009, 64, 229-238.

10. Joo, E.J.; Kang, C.I.; Ha, Y.E.; Kang, S.J.; Park, S.Y.; Chung, D.R.; Peck, K.R.; Lee, N.Y.; Song, J.H. Risk factors for mortality in patients with Pseudomonas aeruginosa bacteremia: Clinical impact of antimicrobial resistance on outcome. Microb. Drug Resist. 2011, 17, 305-312. 
11. Wright, H.; Bonomo, R.A., Paterson, D.L. New agents for the treatment of infections with Gramnegative bacteria: restoring the miracle or false dawn? Clin. Microbiol. Infect. 2017, 23, 704-712.

12. Quinn, M.T.; Parthasarathy, S.; Steinberg, D. Lysophosphatidylcholine: A chemotactic factor for human monocytes and its potential role in atherogenesis. Proc. Natl. Acad. Sci. USA. 1988, 85, 2805-2809.

13. Sakai, M.; Miyazaki, A.; Hakamata, H.; Sasaki, T.; Yui, S.; Yamazaki, M.; Shichiri, M.; Horiuchi, S. Lysophosphatidylcholine plays an essential role in the mitogenic effect of oxidized low density lipoprotein on murine macrophages. J. Biol. Chem. 1994, 269, 31430-31435.

14. Liu, Z.; Qin, H.; Yang, Z.; Xia, Y.; Liu, W.; Yang, J.; Jiang, Y.; Zhang, H.; Yang, Z.; Wang, Y.; Zheng, Q. Randomised clinical trial: The effects of perioperative probiotic treatment on barrier function and postoperative infectious complications in colorectal cancer surgery - A double-blind study. Aliment. Pharmacol. Ther. 2011, 33(1), 50-63.

15. Smani, Y.; Domínguez-Herrera, J.; Ibáñez-Martínez, J.; Pachón, J. Therapeutic efficacy of lysophosphatidylcholine in severe infections caused by Acinetobacter baumannii. Antimicrob. Agents Chemother. 2015, 59, 3920-3924.

16. Parra Millán, R.; Jiménez Mejías, M.E.; Sánchez Encinales, V.; Ayerbe Algaba, R.; Gutiérrez Valencia, A.; Pachón Ibáñez, M.E.; Díaz, C.; Pérez Del Palacio, J.; López Cortés, L.F.; Pachón, J.; Smani, Y. Efficacy of lysophosphatidylcholine in combination with antimicrobial agents against Acinetobacter baumannii in experimental murine peritoneal sepsis and pneumonia models. Antimicrob. Agents Chemother. 2016, 60, 4464-4470.

17. Jacqueline, C.; Roquilly, A.; Desessard, C.; Boutoille, D.; Broquet, A.; Le Mabecque, V.; Amador, G.; Potel, G.; Caillon, J.; Asehnoune, K. Efficacy of ceftolozane in a murine model of Pseudomonas aeruginosa acute pneumonia: In vivo antimicrobial activity and impact on host inflammatory response. J. Antimicrob. Chemother. 2013; 68(1), 177-183.

18. Nemzek, J.A.; Hugunin, K.M.S.; Opp, M.R. Modeling sepsis in the laboratory: Merging sound science with animal well-being. Comp. Med. 58, 120-128.

19. Miyazaki, H.; Midorikawa, N.; Fujimoto, S.; Miyoshi, N.; Yoshida, H.; Matsumoto, T. Antimicrobial effects of lysophosphatidylcholine on methicillin-resistant Staphylococcus aureus. Ther. Adv. Infect. Dis. 2017, 4, 89-94.

20. Yan, J.J.; Jung, J.S.; Lee, J.E.; Lee, J.; Huh, S.O.; Kim, H.S.; Jung, K.C.; Cho, J.Y.; Nam, J.S.; Suh, H.W.; Kim, Y.H.; Song, D.K. Therapeutic effects of lysophosphatidylcholine in experimental sepsis. Nat. Med. 2004, 10, 161-167.

21. Quan, H.; Hur, Y.H.; Xin, C.; Kim, J.M.; Choi, J.I.; Kim, M.Y.; Bae, H.B. Stearoyl lysophosphatidylcholine enhances the phagocytic ability of macrophages through the AMP-activated protein kinase/p38 mitogen activated protein kinase pathway. Int. Immunopharmacol. 2016; 39:328-334.

22. Mechkarska, M.; Prajeep, M.; Radosavljevic, G.D.; Jovanovic, I.P.; Al Baloushi, A.; Sonnevend, A.; Lukic, M.L.; Conlon, J.M. An analog of the host-defense peptide hymenochirin-1B with potent broadspectrum activity against multidrug-resistant bacteria and immunomodulatory properties. Peptides 2013, 50, 153-159.

23. Maisetta, G.; Batoni, G.; Esin, S.; Florio, W.; Bottai, D.; Flavilli, F.; et al. In vitro bactericidal activity of human $\beta$-defensin 3 against multidrug-resistant nosocomial strains. Antimicrob. Agents Chemother. 2006, 50, 806-809.

24. Routsias, J. G.; Karagounis, P.; Parvulesku, G.; Legakis, N.J.; Tsakris, A. In vitro bactericidal activity of human $\beta$-defensin 2 against nosocomial strains. Peptides 2010, 31, 1654-1660. 
25. Supp, D.M.; Gardner, J.; Klingenberg, J.M.; Neely, A.N. Antibiotic resistance in clinical isolates of Acinetobacter baumannii, Pseudomonas aeruginosa, and Staphylococcus aureus does not impact sensitivity to human beta defensin 4. Burns 2009, 35, 949-955.

26. Bals, R.; Wang, X.; Zasloff, M.; Wilson, J.M. The peptide antibiotic LL-37/hCAP-18 is expressed in epithelia of the human lung where it has broad antimicrobial activity at the airway surface. Proc. Natl. Acad. Sci. US.A. 1998, 95(16), 9541-9546.

27. De La Fuente-Núñez, C.; Korolik, V.; Bains, M.; Nguyen, U.; Breidenstein, E.B.; Horsman, S.; Lewenza, S.; Burrows, L.; Hancock, R.E. Inhibition of bacterial biofilm formation and swarming motility by a small synthetic cationic peptide. Antimicrob. Agents Chemother. 2012, 56(5), 2696-2704.

28. Cheng, A.C.; Cheng, A.C.; Stephens, D.P.; Currie, B.J. Granulocyte-colony stimulating factor (G-CSF) as an adjunct to antibiotics in the treatment of pneumonia in adults. Cochrane Database Syst. Rev. 2007, 2, CD004400.

29. Blum, C.A.; Nigro, N.; Briel, M.; Schuetz, P.; Ullmer, E.; Suter-Widmer, I.; Winzeler, B.; Bingisser, R.; Elsaesser, H.; Drozdov, D.; Arici, B.; Urwyler, S.A.; Refardt, J.; Tarr, P.; Wirz, S.; Thomann, R.; Baumgartner, C.; Duplain, H.; Burki, D.; Zimmerli, W.; Rodondi, N.; Mueller, B.; Christ-Crain, M. Adjunct prednisone therapy for patients with community-acquired pneumonia: A multicentre, doubleblind, randomised, placebo-controlled trial. Lancet 2015, 385, 1511-1518.

30. Peña, C.; Suarez, C.; Gonzalo, M.; Murillas, J.; Almirante, B.; Pomar, V.; Aguilar, M.; Granados, A.; Calbo, E.; Rodríguez-Baño, J.; Rodríguez, F.; Tubau, F.; Martínez-Martínez, L.; Oliver, A. Prospective multicenter study of the impact of carbapenem resistance on mortality in Pseudomonas aeruginosa bloodstream infections. Antimicrob. Agents Chemother. 2012, 56, 1265-1272.

31. Clinical and Laboratory Standards Institute. Performance Standards for Antimicrobial Susceptibility Testing. wenty-Fourth Informational Supplement. Document M100-S24. CLSI, Wayne, PA, 2014.

32. Smani, Y.; López-Rojas, R.; Domínguez-Herrera, J.; Docobo-Pérez, F.; Martí, S; Vila, J.; Pachón, J. In vitro and in vivo reduced fitness and virulence in ciprofloxacin-resistant Acinetobacter baumannii. Clin. Microbiol. Infect. 2012, 18, E1-4.

33. National Research Council. Guide for the Care and Use of Laboratory Animals: Eighth Edition. National Academies Press, Washington, DC, 2011.

34. Ye, G.; Cai, X.; Wang, B.; Zhou, Z.; Yu, X.; Wang, W.; Zhang, J.; Wang, Y.; Dong, J.; Jiang, Y. Simultaneous determination of vancomycin and ceftazidime in cerebrospinal fluid in craniotomy patients by high-performance liquid chromatography. J. Pharm. Biomed. Anal. 2008, 48, 860-865.

35. Usansky JL, Desai A, T.-L. D. PK Functions for Microsoft Excel, Allergan, Irvine, CA: Department of Pharmacokinetics and Drug Metabolism; USA. https://www.boomer.org/pkin/soft.html, 2012.

36. Craig, W. Pharmacokinetic/pharmacodynamic parameters: rationale for antibacterial dosing of mice and men. Clin. Infect. Dis. 1998, 26, 1-10.

37. Pachón-Ibáñez, M. E.; Docobo-Pérez, F.; López-Rojas, R.; Domínguez-Herrera, J.; Jiménez-Mejías, M.E.; Garcia-Curiel, A.; Pichardo, C.; Jiménez, L.; Pachón, J. Efficacy of rifampin and its combinations with imipenem, sulbactam, and colistin in experimental models of infection caused by imipenem-resistant Acinetobacter baumannii. Antimicrob. Agents Chemother. 2010, 54, 1165-1172. 